\title{
New chronic disease medication prescribing by nurse practitioners, physician assistants, and primary care physicians: a cohort study
}

\author{
Zachary A. Marcum ${ }^{1 *}$, Johanna E. Bellon ${ }^{2}$, Jie Li ${ }^{3}$, Walid F. Gellad ${ }^{4}$ and Julie M. Donohue ${ }^{3}$
}

\begin{abstract}
Background: Medications to treat and prevent chronic disease have substantially reduced morbidity and mortality; however, their diffusion has been uneven. Little is known about prescribing of chronic disease medications by nurse practitioners (NPs) and physician assistants (PAs), despite their increasingly important role as primary care providers. Thus, we sought to conduct an exploratory analysis to examine prescribing of new chronic disease medications by NPs and PAs compared to primary care physicians (PCPs).

Methods: We obtained prescribing data from IMS Health's Xponent ${ }^{\text {TM }}$ on all NPs, PAs, and PCPs in Pennsylvania regularly prescribing anticoagulants, antihypertensives, oral hypoglycemics, and/or HMG-Co-A reductase inhibitors pre- and post-introduction of five new drugs in these classes that varied in novelty (i.e., dabigatran, aliskiren, sitagliptin or saxagliptin, and pitavastatin). We constructed three measures of prescriber adoption during the 15-month post-FDA approval period: 1) any prescription of the medication, 2) proportion of prescriptions in the class for the medication, and 3) time to adoption (first prescription) of the medication.

Results: From 2007 to 2011, the proportion of antihypertensive prescriptions prescribed by NPs and PAs approximately doubled from 2.0 to $4.2 \%$ and 2.2 to $4.9 \%$, respectively. Similar trends were found for anticoagulants, oral hypoglycemics, and HMG-Co-A reductase inhibitors. By 2011, more PCPs had prescribed each of the newly approved medications than NPs and PAs (e.g., $44.3 \%$ vs. $18.5 \%$ vs. $20 \%$ for dabigatran among PCPs, NPs, and PAs). Across all medication classes, the newly approved drugs accounted for a larger share of prescriptions in the class for PCPs followed by PAs, followed by NPs (e.g., dabigatran: $4.9 \%$ vs. $3.2 \%$ vs. $2.8 \%$, respectively). Mean time-to-adoption for the newly approved medications was shorter for PCPs compared to NPs and PAs (e.g., dabigatran, 7.3 vs. 8.2 vs. 8.5 months; $P$ all medications $<0.001$ ).

Conclusions: PCPs were more likely to prescribe each of the newly approved medications per each measure of drug adoption, regardless of drug novelty. Differences in the rate and speed of drug adoption between PCPs, NPs, and PAs may have important implications for care and overall costs at the population level as NPs and PAs continue taking on a larger role in prescribing.
\end{abstract}

Keywords: Health services research, Pharmacoepidemiology, Primary care, Work force

\footnotetext{
* Correspondence: zmarcum@uw.edu

'Department of Pharmacy, School of Pharmacy, University of Washington,

1959 NE Pacific St, Box 357630, Seattle, WA 98102, USA

Full list of author information is available at the end of the article
} 


\section{Background}

Since the 1977 passage of the Rural Health Clinic Services Act, nurse practitioners (NPs) and physician assistants (PAs) have been authorized by Medicare and Medicaid to be reimbursed as providers [1]. Although all states allow NPs and PAs to practice in some capacity, scope-ofpractice laws vary widely by state [2, 3]. Nationally, approximately $56 \%$ of physician group practices and $40 \%$ of independent providers utilize NPs and PAs in their practices, with continued growth expected due to a confluence of factors - fewer medical students choosing primary care, increasing health care demand from a growing and aging population, and a broad increase in access to care through the Affordable Care Act $[1,4,5]$. NPs and PAs generally perform $80 \%$ of the functions of physicians, and studies to-date indicate that their quality measures and patient satisfaction are equal or greater than physicians' for care within their scope-of-practice [1, 6-8].

One of the most important roles of a primary care provider - NP, PA, or primary care physician (PCP) - is to manage chronic diseases, and pharmacotherapy is the mainstay of treatment. National estimates for the US indicate that approximately $70 \%$ of primary care office visits result in a medication being prescribed [9]. A review of state NP and PA regulations from 2001 to 2010 found that most states have loosened regulations over time, granting greater autonomy to NPs and PAs, particularly with respect to prescriptive authority [10].

Some of the most widely prescribed chronic medication classes are those to treat hyperlipidemia and prevent the development and progression of ischemic heart disease, stroke, diabetes, and heart failure, which have greatly reduced cardiovascular disease-related morbidity and mortality $[11,12]$. However, diffusion of these therapies across the US is sub-optimal. There is both 'under-diffusion' of evidence-based therapies and 'over-diffusion' of new drugs that are more costly but no more effective than existing therapies. Diffusion is known to vary by physician specialty, with PCPs typically adopting new drugs more slowly than physicians with sub-specialty training [13]. However, it is not known how NPs and PAs utilize newlyapproved drugs compared to PCPs despite their increasingly important role as primary care providers. Differential uptake of new chronic disease medications by NPs and PAs compared to physicians in primary care has potential implications for both cost and quality of care. Thus, we sought to conduct an exploratory analysis to examine the prescribing of new cardiovascular and diabetes medications by NPs and PAs relative to that of PCPs.

\section{Methods}

Data sources and sample

We used IMS Health's Xponent ${ }^{\mathrm{Tm}}$ prescription database to characterize NP, PA, and PCP prescribing of five new chronic disease medications (representing four medication classes), and IMS Healthcare Organization Services ${ }^{\mathrm{TM}}$ (HCOS) database for provider specialty and organizational affiliations. While data exist for physician characteristics, such as age, medical school, and residency program, in the American Medical Association Physician Masterfile, an equivalent dataset does not exist for NPs and PAs. As a result, we were unable to measure these provider-level characteristics in our sample. Xponent ${ }^{\mathrm{Tm}}$ directly captures $86 \%$ of all US prescriptions filled in retail pharmacies and utilizes a patented proprietary projection method to represent $100 \%$ of prescriptions filled in these outlets [14]. We obtained monthly provider-level data on all prescriptions of interest dispensed in Pennsylvania between January 1, 2007 and December 31, 2011. Of note, certified registered NPs and PAs in Pennsylvania are both authorized to prescribe medical and therapeutic treatments. Xponent ${ }^{\mathrm{TM}}$ contains limited patient-level information, including the source of payment (Medicare, Medicaid fee-for-service, commercial insurance, cash or uninsured) and patient age, with no individual patient identifiers. We obtained information on provider specialty, provider sex, and organizational affiliations (e.g., medical group) from IMS Health's HCOS database. HCOS data were used to identify primary care medical groups and to specify the number of providers within each medical group.

\section{Study cohort}

We compiled data for four cohorts of prescribers - one for each of the medication classes under investigation - including prescribers of oral anticoagulants, antihypertensives targeting the renin-angiotensin-aldosterone system, oral hypoglycemics, and HMG-CoA reductase inhibitors among the three prescriber types (i.e., NPs, PAs, and PCPs). We excluded those who never prescribed a medication from the class of interest during the study period. Because we were interested in primary care prescribing behavior, we excluded those providers not practicing in a primary care practice as well as those with missing values for National Provider Identifier or sex.

\section{Study medications}

We measured new medication adoption in our sample for each of five new medications in the four classes of interest (dabigatran, FDA approval: 10/2010; aliskiren, approved 3/2007; sitagliptin and saxagliptin, approved $10 / 2006$ and $7 / 2009$, respectively; and pitavastatin approved $8 / 2009)$. These drugs were of varying novelty as assessed by their benefit/risk profiles and mechanisms of action - and differed in their order of entry in their respective therapeutic classes. For example, dabigatran was the first of a new class of oral anticoagulants with improved efficacy and potentially lower risk of bleeding 
compared to warfarin in patients with atrial fibrillation $[15,16]$. Sitagliptin and saxagliptin were part of a new class of oral hypoglycemic agents that target a different physiologic pathway than other diabetes medications and were purported to have minimal risk of hypoglycemia and weight gain compared to sulfonylureas with comparable efficacy [17]. Aliskiren was the first in a new class of agents for hypertension, joining two others (i.e., angiotensin-converting enzyme inhibitors and angiotensin II receptor blockers) that inhibit the renin-angiotensin-aldosterone system; comparative efficacy and safety among the antihypertensive classes is largely unknown. Pitavastatin was the seventh 'statin' approved in the US and represents the most minor therapeutic advance among the study drugs [18]. This variation in medication classes and novelty allowed us to assess whether any differences between NP, PA, and PCP prescribing were specific to a medication class or consistent across multiple classes.

\section{Outcome measures}

The decision to adopt a new drug is multifaceted. A prescriber first needs to learn about a new drug and then decide whether to use it and how frequently to prescribe it. Therefore, we constructed three measures: 1) any prescription of the newly approved medication in the final year of the study period $(2011), 2$ ) proportion of new medication among the medication class, and 3) time to first adoption of the newly approved medication. Time to first adoption - defined as the time from FDA approval to first prescription of the new drug - provides a measure of speed of adoption, while the other two measures identify the extent of provider adoption over time [19]. In order to assess NP and PA prescribing trends in general, we also measured the share of prescribing within the drug classes of interest accounted for by NPs and PAs compared to PCPs over time.

\section{Statistical analysis}

Our analysis followed three steps. First, for each of the four chronic disease medication classes, we described characteristics of NPs, PAs, and PCPs who were regular prescribers of the medication class as a frequency (percentage) for each variable. We used Chi-square tests to assess differences in characteristics across provider types, and Wilcoxon rank sum tests for non-normally distributed variables. Second, for each of the four chronic disease medication classes, we measured the proportion of all medications within the class prescribed by the three provider types (i.e., NPs, PAs, and PCPs) across all years. Third, we estimated new drug adoption among the three provider types by assessing the three measures of adoption previously described. To assess time to first adoption, we used the Kaplan-
Meier method to compute the proportion of providers who had adopted the new drug in the 15 months postFDA approval. The date of first prescription of each newly approved medication in the dataset was used as the index date.

Given evidence suggesting the potential for sex differences in new drug adoption by providers [20], we conducted post-hoc sensitivity analyses. Because sex was almost perfectly correlated with provider type, we could not control for it in multivariable analysis. Thus, we repeated all primary analyses described above on only female providers across the three provider types. We used SAS version 9.3 (SAS Institute, Cary, NC, USA) for analyses and the Stata version 11.0 (StataCorp, College Station, TX, USA) for the Kaplan-Meier graphs.

\section{Results}

\section{Characteristics of the study sample}

We identified more than 5000 NPs, PAs, and PCPs in Pennsylvania who prescribed each chronic disease medication category in 2007-2011 (oral anticoagulants, $n=$ 5299; select antihypertensives, $n=5514$; oral hypoglycemics, $n=5510$; HMG-CoA reductase inhibitors, $n=5454$; and all four classes, $n=5177)$. Among the medication classes with the most prescribers - antihypertensives NPs and PAs were more likely than PCPs to be female (93\% of NPs and $77 \%$ of PAs vs. $34 \%$ of PCPs, $P<0.001$ ) (Table 1). NPs and PAs were also more than twice as likely to practice in a rural setting (21\% of NPs and $27 \%$ of PAs vs. $12 \%$ of PCPs, $P<0.001)$. PCPs were slightly more likely to prescribe to older patients (with age $\geq 65$ years representing $52 \%$ of PCP patients vs. $41 \%$ of NP patients and $40 \%$ of PAs, $P<0.001)$. PCPs prescribed a larger annual volume of antihypertensive prescriptions compared to NPs and PAs (2011 mean: PCPs, 1127.2; NPs, 433.3; PAs, 423.8; $P<0.001)$. Similar results were found for the other three medication classes (see Additional file 1: Tables S1-S3).

\section{Prescribing patterns over time}

From 2007 to 2011, the proportion of all prescriptions in these classes written by NPs and PAs increased substantially. Among select antihypertensives, the proportion of prescriptions written by NPs and PAs approximately doubled from 2.0 to $4.2 \%$ and 2.2 to $4.9 \%$ among NPs and PAs, respectively (Table 2). Conversely, the proportion of all antihypertensive prescriptions accounted for by PCPs decreased from 95.9 to $91.0 \%$ (Table 2). Similar results were found for the other three medication classes.

\section{New drug adoption}

By the final year of the study period (2011), more PCPs had prescribed each of the newly approved chronic 
Table 1 Characteristics of nurse practitioners, physician assistants, and primary care physicians who prescribed select antihypertensive ${ }^{\mathrm{a}}$ prescription medications in Pennsylvania, 2011 ${ }^{\mathrm{b}}$

\begin{tabular}{|c|c|c|c|}
\hline \multirow[t]{2}{*}{ Characteristics } & $\begin{array}{l}\text { Nurse } \\
\text { practitioner }\end{array}$ & $\begin{array}{l}\text { Physician } \\
\text { assistant }\end{array}$ & $\begin{array}{l}\text { Primary care } \\
\text { physician }\end{array}$ \\
\hline & $N=504$ & $N=591$ & $N=4419$ \\
\hline Provider sex, \% & - & - & - \\
\hline Female & 93.1 & 76.8 & 34.2 \\
\hline Provider setting, $\%$ & - & - & - \\
\hline Rural & 20.8 & 27.4 & 11.9 \\
\hline Provider prescribing & - & - & - \\
\hline $\begin{array}{l}\text { Annual prescription } \\
\text { volume, mean (sd) }\end{array}$ & $433.3(496.6)$ & $423.8(496.0)$ & $1127.2(853.8)$ \\
\hline Patient age, \% & - & - & - \\
\hline$<64$ & 59.1 & 60.0 & 47.7 \\
\hline $65-74$ & 19.9 & 20.2 & 22.6 \\
\hline $75-84$ & 13.4 & 13.0 & 18.5 \\
\hline $85+$ & 7.7 & 6.8 & 11.2 \\
\hline $\begin{array}{l}\text { Source of payment } \\
\text { for prescription, } \%\end{array}$ & - & - & - \\
\hline Cash & 5.3 & 5.5 & 5.3 \\
\hline Commercial & 55.2 & 54.8 & 54.9 \\
\hline Medicaid & 8.7 & 9.1 & 5.4 \\
\hline Medicare & 30.7 & 30.6 & 34.3 \\
\hline $\begin{array}{l}\text { Primary care medical } \\
\text { group }\end{array}$ & - & - & - \\
\hline Number of practice sites & 550 & 569 & 3277 \\
\hline $\begin{array}{l}\text { Number of providers per } \\
\text { site, mean (sd) }\end{array}$ & $45.1(160.1)$ & $47.2(148.9)$ & $20.7(89.0)$ \\
\hline
\end{tabular}

${ }^{a}$ Antihypertensive medications included those targeting the renin-angiotensinaldosterone system, including angiotensin-converting enzyme inhibitors, angiotensin II receptor blockers, and direct renin inhibitors; ${ }^{\mathrm{b}} \mathrm{P}<0.001$ for comparisons across providers for all variables

disease medications than NPs and PAs (Fig. 1; Table 3). For example, $44.3 \%$ of PCPs ordered at least one prescription for dabigatran compared to $18.5 \%$ of NPs and $20.0 \%$ of PAs (Fig. 1). We found similar differences for each of the other new medications $(31.9 \%$ vs. $13.9 \%$ vs. $20.4 \%$ for aliskiren among PCPs, NPs, and PAs; $87.3 \%$ vs. $71.5 \%$ vs. $72.5 \%$ for sitagliptin or saxagliptin among PCPs, NPs, and PAs; and $17.1 \%$ vs. $6.9 \%$ vs. $12.0 \%$ for pitavastatin among PCPs, NPs, and PAs). Across all medication classes, the new cardiovascular agents accounted for a larger share of all prescriptions in the class for PCPs followed by PAs, followed by NPs (Table 3). For example, dabigatran prescriptions accounted for $4.9 \%$ of all anticoagulant prescriptions ordered by PCPs compared to $3.2 \%$ for PAs and $2.8 \%$ for NPs. Similarly, PCPs adopted each of the newly approved cardiovascular medications significantly more rapidly than did NPs and PAs during the post-FDA
Table 2 Proportion of cardiovascular prescriptions ordered by nurse practitioners, physician assistants, and primary care physicians over time, 2007-2011

\begin{tabular}{llllll}
\hline & 2007 & 2008 & 2009 & 2010 & 2011 \\
\hline $\begin{array}{c}\text { Antihypertensives } \\
\begin{array}{c}\text { Total } \\
\text { prescriptions, N }\end{array}\end{array}$ & $2,116,048$ & $2,182,069$ & $2,188,136$ & $2,248,183$ & $2,272,489$ \\
NP (\%) & 2.0 & 2.4 & 3.0 & 3.8 & 4.2 \\
PA (\%) & 2.2 & 2.8 & 3.4 & 4.2 & 4.9 \\
PCP (\%) & 95.9 & 94.8 & 93.6 & 92.1 & 91.0 \\
Anticoagulants & & & & & \\
Total, N & 261,698 & 264,176 & 267,630 & 278,745 & 291,524 \\
NP & 1.5 & 1.9 & 2.3 & 3.2 & 3.4 \\
PA & 1.4 & 2.1 & 2.8 & 3.6 & 4.1 \\
PCP & 97.1 & 96.0 & 94.9 & 92.6 & 92.6
\end{tabular}

Oral hypoglycemics

$\begin{array}{llllll}\text { Total, N } & 1,292,094 & 1,293,618 & 1,302,716 & 1,315,114 & 1,301,535 \\ \text { NP } & 1.9 & 2.3 & 2.9 & 3.7 & 4.0 \\ \text { PA } & 2.1 & 2.7 & 3.3 & 4.1 & 4.8 \\ \text { PCP } & 96.0 & 95.0 & 93.8 & 92.3 & 91.2\end{array}$

HMG-CoA Reductase inhibitors

\begin{tabular}{llllll} 
Total, $N$ & $1,294,269$ & $1,319,042$ & $1,359,293$ & $1,411,180$ & $1,455,255$ \\
NP & 2.1 & 2.6 & 3.3 & 4.1 & 4.4 \\
PA & 2.3 & 2.8 & 3.5 & 4.4 & 5.1 \\
PCP & 95.6 & 94.5 & 93.2 & 91.6 & 90.5 \\
\hline
\end{tabular}

Abbreviations: NP nurse practitioner, $P A$ physician assistant, $P C P$ primary care physician

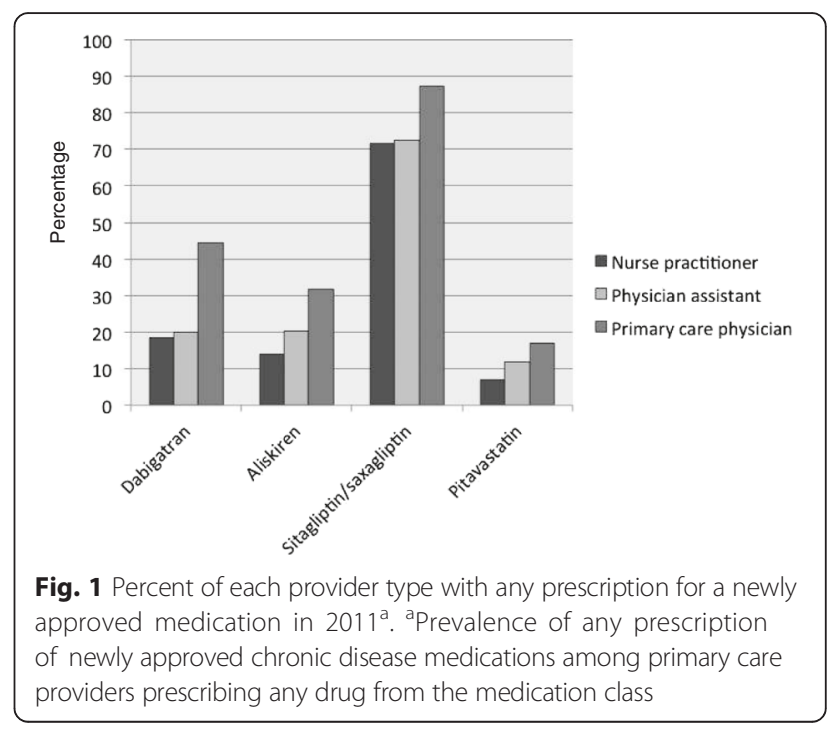


Table 3 Proportion of prescriptions in each category accounted for by the newly approved medications, 2011

\begin{tabular}{ll}
\hline Primary care provider type $^{\text {a }}$ & $\begin{array}{l}\text { Total number of prescriptions, } N \text { (\% of } \\
\text { all prescriptions in medication class })\end{array}$ \\
\hline Nurse practitioner $(N=383)$ & $280(2.8)$ \\
Physician assistant $(N=451)$ & $374(3.2)$ \\
Primary care physician $(N=4148)$ & $13,261(4.9)$ \\
& Aliskiren \\
Nurse practitioner $(N=469)$ & $478(0.5)$ \\
Physician assistant $(N=548)$ & $707(0.6)$ \\
Primary care physician $(N=4338)$ & $16,793(0.8)$ \\
Nurse practitioner $(N=435)$ & Sitagliptin/saxagliptin \\
Physician assistant $(N=527)$ & $7895(11.7)$ \\
Primary care physician $(N=4312)$ & $153,926(13.0)$ \\
Nurse practitioner $(N=451)$ & Pitavastatin \\
Physician assistant $(N=526)$ & $299(0.4)$ \\
Primary care physician $(N=4299)$ & $6367(0.5)$ \\
\hline
\end{tabular}

${ }^{a} \mathrm{~N}$ indicates the total number of each provider type regularly prescribing each medication class

approval period (Fig. 2; $P<0.001$ ). Mean time-to-adoption for the newly approved medications was shorter for PCPs compared to NPs and PAs: dabigatran, 7.3 vs. 8.2 vs. 8.5 months; aliskiren, 6.6 vs. 9.0 vs. 7.9 months; sitagliptin, 4.4 vs. 5.7 vs. 6.9 ; saxagliptin, 8.4 vs. 10.1 vs. 9.7 ; and pitavastatin, 7.6 vs. 8.8 vs. 9.5 .

\section{Sensitivity analyses: only female providers}

When sub-setting the analyses to only female providers, the results were largely unchanged - female PCPs adopted the new drugs more rapidly than did NPs and PAs (Additional file 1: Tables S4-S6, Figures S1 and S2). However, there were some noteworthy differences between the main analysis and the sensitivity analysis. Across the included medication classes, the proportion of prescriptions written by female NPs and PAs was greater than in the main analysis (Additional file 1: Table S5). In addition, consistent with the main analysis, by the final year of the study period (2011), dabigatran accounted for a larger share of all prescriptions in the class for female PCPs followed by female PAs, followed by female NPs (Additional file 1: Table S6). In contrast to the main analysis, this pattern did not follow for aliskiren, sitagliptin/saxagliptin, or pitavastatin, for which female PAs accounted for the largest share of prescriptions of the new medications. By the final year of the study period (2011), more female PCPs had prescribed each of the newly approved chronic disease medications than female NPs and PAs, with the exception of pitavastatin (Additional file 1: Figure S1). Similarly, female PCPs adopted each of the newly approved cardiovascular medications significantly more rapidly than did female NPs and PAs during the post-FDA approval period (Additional file 1: Figure S2; $P<0.01$ ).

\section{Discussion}

Our exploratory study yielded three key findings regarding NP, PA, and PCP prescribing of newly approved chronic disease medications in the primary care setting. First, we found that NPs and PAs ordered a small but increasing share of prescriptions in some of the most widely used classes, responsible for almost 1 in 10 prescriptions in these classes by 2011. Second, we found that PCPs adopted each of the new chronic disease medications more rapidly than did NPs and PAs, regardless of drug novelty. Third, we found that PCPs ordered a greater proportion of their prescriptions for the new medications than did NPs and PAs. Differences in the rate and speed of drug adoption between PCPs and NPs and PAs may have important implications for care and overall costs at the population level as NPs and PAs continue to take on a larger role in prescribing.

To our knowledge, this study is the first to examine new drug adoption across primary care provider types. This is noteworthy because much of the existing literature comparing NPs and PAs and PCPs has focused on process measures (e.g., patient satisfaction), with less emphasis on utilization measures. For example, a systematic review comparing NPs to PCPs found that processes of care were not significantly different between the two provider types for chronic disease management [21]. Moreover, a Cochrane Review evaluating the impact of doctor-nurse substitution in primary care on patient outcomes, processes of care, and resource utilization reported that appropriately trained NPs can produce as high quality care as PCPs on the measured processes and achieve comparable health outcomes [7]. However, the review acknowledged major limitations in the current literature, including only one study being powered to assess equivalence of care, several studies having methodological limitations, and patient follow-up being short. [7] The most comparable study to ours (not included in the Cochrane Review) examined use of diagnostic imaging ordered by NPs and PAs relative to that of PCPs following office-based encounters as a measure of healthcare utilization. Hughes et al. found that NPs and PAs ordered more imaging services than PCPs for similar patients and concluded that although these differences appeared modest at the individual level, the potential implications on care and costs at the population level are significant [5]. Prior research from the National Ambulatory Medical Care Surveys found that NPs and PAs 


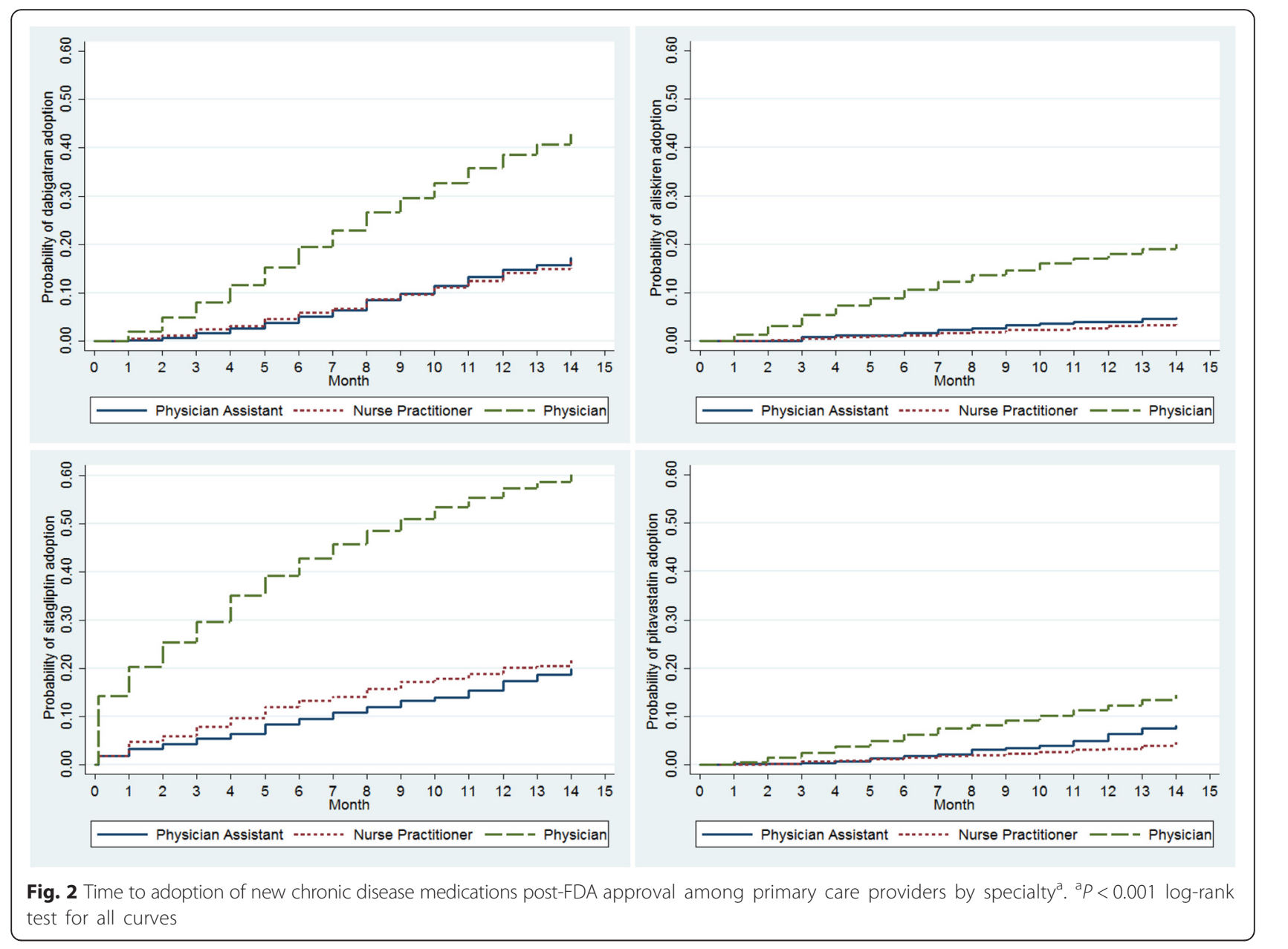

and PCPs prescribe a similar number of medications per visit [4]. However, our study revealed differential prescribing behavior between NPs and PAs and PCPs for newly approved drugs.

The differences in prescribing we identify may be due to patient-, practice-, provider-, manufacturer-, or payerlevel factors that differ between provider types. Previous research has shown that NPs and PAs generally spend greater amounts of time educating and counseling patients and that physicians retain older, more complex patients [22]. Our findings are consistent in that PCPs were more likely than NPs and PAs to prescribe to older patients, who often take multiple medications due to chronic co-morbidity. Given an older, more complex patient panel, PCPs may be more likely to prescribe from a broader prescription armamentarium, including newly approved drugs. In addition, some evidence points to provider sex as an important factor in health technology adoption; male providers tend to adopt new treatment more rapidly on average [19]. Our findings support this notion, given that the vast majority of NPs and PAs were female compared to one-third of PCPs. To explore this, we conducted post-hoc sensitivity analyses by including only female NPs, PAs, and PCPs in order to qualitatively compare these results with the main results. These results were largely similar to the main results, with a few exceptions. On the whole, we continued to find statistically significant differences in the rate of new drug adoption across provider type. Further, differences in peer influences, which may affect new technology adoption, could differ between NPs, PAs, and PCPs [23-25]. It is interesting to note, however, that NPs and PAs in our study worked in much larger practices where there are presumably more peers to learn from and, yet, they were found to be slower adopters of new drugs. Another factor that could drive new drug adoption is pharmaceutical marketing. However, previous research has shown that NPs have similar exposure to pharmaceutical promotion and tend to respond in the same way as physicians [4]. We also note that there were differences in speed and extent of adoption across the five study drugs, with first-in-class drugs being more rapidly adopted than others. Finally, we found that NPs and PAs were more likely to prescribe to patients receiving Medicaid 
coverage, and it is possible that Medicaid formularies were more restrictive, thus explaining a lower use of new brand name drugs.

Our study has important limitations. First, we lacked clinical information on patient-level factors (e.g., comorbidities). Prescriber adoption decisions may be influenced by their patient case mix although prior studies suggest this explains little of the variation in choice of prescription drugs [26]. Second, we lacked data on the provider characteristics, such as age, training, and years in practice. As previously mentioned, data exist for physician characteristics (e.g., age, sex, medical school, and residency program) in the American Medical Association Physician Masterfile; however, an equivalent dataset does not exist for NPs and PAs. As a result, we were unable to measure these provider-level characteristics. Third, because of a lack of data, we were unable to adjust for some of the external influences on prescribing behavior, such as manufacturer promotional efforts directed at providers, characteristics of the specific organizations in which providers practice, and health plan coverage of other medications. Fourth, our analysis included five newly approved medications from four medication classes and, thus, may not represent prescribing habits of primary care providers for all newly approved medications. Finally, we were not able to assess appropriateness for the prescribing behaviors and thus cannot say whether there is underuse of new drugs by NPs and PAs and/or overuse by PCPs.

\section{Conclusions}

In conclusion, our exploratory findings point to differential adoption of newly approved chronic disease medications between NPs and PAs and PCPs. PCPs were more likely to prescribe each of the newly approved medications, including both more and less novel drugs, by all three measures of adoption used in our study. While there were small absolute differences among prescriber groups detected, these may have important implications for care and overall costs at the population level given the number of new drugs approved each year.

\section{Additional file}

Additional file 1: Table S1. Characteristics of nurse practitioners, physician assistants, and primary care physicians who prescribed anticoagulant prescription medications in Pennsylvania, 2011. Table S2. Characteristics of nurse practitioners, physician assistants, and primary care physicians who prescribed oral hypoglycemic prescription medications in Pennsylvania, 2011. Table S3. Characteristics of nurse practitioners, physician assistants, and primary care physicians who prescribed HMG-CoA reductase inhibitor prescriptions in Pennsylvania, 2011. Table S4. Characteristics of female nurse practitioners, physician assistants, and primary care physicians who prescribed select antihypertensive prescription medications in Pennsylvania, 2011. Table S5. Proportion of cardiovascular prescriptions ordered by female nurse practitioners, physician assistants, and primary care physicians over time, 2007-2011. Table S6. Proportion of prescriptions from female providers in each category accounted for by the newly approved medications, 2011. Figure S1. Percent of each female provider type with any prescription for a newly approved medication in 2011. Figure S2. Time to adoption of new chronic disease medications post-FDA approval among female primary care providers. (DOCX $168 \mathrm{~kb}$ )

\section{Abbreviations}

HCOS, Healthcare Organization Services; NP, nurse practitioner; PA, physician assistant; PCP, primary care physician

\section{Acknowledgements}

Not applicable.

\section{Funding}

This work was supported by R01HL119246 from NHLBI (Drs. Donohue and Gellad). Dr. Gellad was also supported by a VA HSR\&D Career Development Award (CDA 09-207). Drs. Donohue and Gellad were partially supported by a pilot grant from the RAND-University of Pittsburgh Health Institute (RUPHI) and Grant Number UL1 RR024153 from the National Center for Research Resources (NCRR), a component of the National Institutes of Health.

\section{Availability of data and materials}

The datasets generated during and/or analysed during the current study are not publicly available due to an ongoing data use agreement but are available from the corresponding author on reasonable request.

\section{Authors' contributions}

ZM participated in the design and interpretation of the data and drafting/ revising the manuscript. JD conceived of the study, and participated in its design and interpretation and helped to draft the manuscript. JL performed the statistical analysis and revised the manuscript critically. JB and WG participated in its design and interpretation and helped to revise the manuscript critically. All authors read and approved the final manuscript.

\section{Competing interests}

None of the authors has relevant competing interests to report. The views of Dr. Gellad represent his alone and are not necessarily representative of the views of the Department of Veterans Affairs or US Government.

Consent for publication

Not applicable.

\section{Ethics approval and consent to participate}

All procedures performed in studies were in accordance with the 1964 Declaration of Helsinki and its later amendments or comparable ethical standards. The University of Pittsburgh Institutional Review Board deemed the study exempt

\section{Author details}

Department of Pharmacy, School of Pharmacy, University of Washington, 1959 NE Pacific St, Box 357630, Seattle, WA 98102, USA. ${ }^{2}$ Wolff Center at UPMC, Ross Building, Room 219, 4601 Baum Blvd, Pittsburgh, PA 15213, USA ${ }^{3}$ Department of Health Policy \& Management, Graduate School of Public Health, University of Pittsburgh, 130 De Soto Street, Pittsburgh, PA 15261, USA. ${ }^{4}$ VA Pittsburgh Healthcare System and Department of General Internal Medicine, School of Medicine, University of Pittsburgh, 200 Meyran Avenue, Parkvale Building, Pittsburgh, PA 15213, USA.

Received: 7 April 2016 Accepted: 20 July 2016

Published online: 27 July 2016

\section{References}

1. Staton FS, Bhosle MJ, Camacho FT, et al. How PAs improve access to care for the underserved. JAAPA. 2007;20(6):32-40.

2. Wing $P$, Langelier $M H$, Salsberg ES, et al. The changing professional practice of physician assistants: 1992 to 2000. JAAPA. 2004;17(1):37-40. 42,45-49.

3. Ricketts TC. Workforce issues in rural areas: a focus on policy equity. Am J Public Health. 2005;95(1):42-8. 
4. Hooker RS, McCaig LF. Use of physician assistants and nurse practitioners in primary care, 1995-1999. Health Aff (Millwood). 2001;20(4):231-8.

5. Hughes DR, Jiang M, Duszak Jr R. A comparison of diagnostic imaging ordering patterns between advanced practice clinicians and primary care physicians following office-based evaluation management visits. JAMA Intern Med. 2015;175(1):101-7.

6. Baldwin KA, Sisk RJ, Watts P, et al. Acceptance of nurse practitioners and physician assistants in meeting the perceived needs of rural communities Public Health Nurs. 1998;15(6):389-97.

7. Laurant $M$, Reeves $D$, Hermens $R$, et al. Substitution of doctors by nurses in primary care. Cochrane Database Syst Rev. 2005;2:CD001271.

8. Halter M, Drennan V, Chattopadhyay K, et al. The contribution of physician assistants in primary care: a systematic review. BMC Health Serv Res. 2013; 13:223. doi:10.1186/1472-6963-13-223.

9. Raofi S, Schappert SM. Medication therapy in ambulatory medical care: United States, 2003-04. National Center for Health Statistics. Vital Health Stat. 2006;13(163):1.

10. Gadbois EA, Miller EA, Tyler D, et al. Trends in state regulation of nurse practitioners and physician assistants, 2001 to 2010. Med Care Res Rev. 2015;72(2):200-19.

11. Ford ES, Ajani UA, Croft JB, et al. Explaining the decrease in U.S. deaths from coronary disease,1980-2000. N Engl J Med. 2007:356(23):2388-98.

12. Wijeysundera HC, Machado M, Farahati F, et al. Association of temporal trends in risk factors and treatment uptake with coronary heart disease mortality, 1994-2005. JAMA. 2010;303(18):1841-7.

13. De Smet BD, Fendrick AM, Stevenson JG, et al. Over and under-utilization of cyclooxygenase-2 selective inhibitors by primary care physicians and specialists: the tortoise and the hare revisited. J Gen Intern Med. 2006; 21(7):694-7.

14. IMS Health. Information Sources, IMS Xponent. http://www.imshealth.com/ portal/site/imshealth/menuitem.c76283e8bf81e98f53c753c71ad8c22a/? vgnextoid=39d15dba9648e310VgnVCM10000076192ca2RCRD\&vgn extchannel=abb6e590cb4dc310VgnVCM100000a48d2ca2RCRD. Accessed 26 September 2015.

15. Connolly SJ, Ezekowitz MD, Yusuf S, et al. Dabigatran versus warfarin in patients with atrial fibrillation. N Engl J Med. 2009;361(12):1139-51.

16. Graham DJ, Reichman ME, Wernecke M, et al. Cardiovascular, bleeding, and mortality risks in elderly Medicare patients treated with dabigatran or warfarin for non-valvular atrial fibrillation. Circulation. 2015;131(2):157-64.

17. Dicker D. DPP-4 inhibitors: impact on glycemic control and cardiovascular risk factors. Diabetes Care. 2011;34 Suppl 2:S276-8.

18. Duggan ST. Pitavastatin: a review of its use in the management of hypercholesterolemia or mixed dyslipidemia. Drugs. 2012;72(4):565-84.

19. Huskamp HA, O'Malley AJ, Horvitz-Lennon M, et al. How quickly do physicians adopt new drugs? The case of second-generation antipsychotics. Psychiatr Serv. 2013;64(4):324-30

20. Lo-Ciganic W, Gellad WF, Huskamp HA, et al. Who were the early adopters of dabigatran? An application of group-based trajectory models. Med Care. 2016;54:725-32.

21. Martínez-González NA, Rosemann T, Tandjung R, et al. The effect of physician-nurse substitution in primary care in chronic diseases: a systematic review. Swiss Med Wkly. 2015;145:w14031.

22. Henry LR, Hooker RS. Retention of physician assistants in rural health clinics. J Rural Health. 2007;23(3):207-14.

23. Menchik DA, Meltzer DO. The cultivation of esteem and retrieval of scientific knowledge in physician networks. J Health Soc Behav. 2010;51(2):137-52.

24. Prosser $\mathrm{H}$, Walley $\mathrm{T}$. New drug prescribing by hospital doctors: the nature and meaning of knowledge. Soc Sci Med. 2006;62(7):1565-78.

25. Prosser H, Almond S, Walley T. Influences on GP's decision to prescribe new drugs-the importance of who says what. Fam Pract. 2003;20(1):61-8.

26. Schneeweiss S, Glynn RJ, Avorn J, et al. A Medicare database review found that physician preferences increasingly outweighed patient characteristics as determinants of first-time prescriptions for COX-2 inhibitors. J Clin Epidemiol. 2005;58(1):98-102.

\section{Submit your next manuscript to BioMed Central and we will help you at every step:}

- We accept pre-submission inquiries

- Our selector tool helps you to find the most relevant journal

- We provide round the clock customer support

- Convenient online submission

- Thorough peer review

- Inclusion in PubMed and all major indexing services

- Maximum visibility for your research

Submit your manuscript at www.biomedcentral.com/submit
Biomed Central 